hep-th/9711124

CU-TP-869

\title{
D-Brane Topology Changing Transitions
}

\author{
Brian R. Greene \\ Departments of Physics and Mathematics \\ Columbia University \\ New York, NY 10025 \\ greene@lnsth1. Ins. cornell. edu
}

\begin{abstract}
We study D-branes on three-dimensional orbifold backgrounds that admit topologically distinct resolutions differing by flop transitions. We show that these distinct phases are part of the vacuum moduli space of the super Yang-Mills gauge theory describing the Dbrane dynamics. In this way we establish that D-branes - like fundamental strings allow for physically smooth topology changing transitions.
\end{abstract}

November 1997 


\section{Introduction}

Spacetime - and the physically accessible transformations which it can undergo is one arena in which string theory has dramatically changed our previous conceptions. The discovery of orbifolds [1] showed us that strings can consistently propagate on mildly singular spaces; $T$-dualities have shown us that strings can probe identical physics while propagating through distinct backgrounds, and the results of [2, 3, 4, have shown us that strings allow the topology of space to change in a physically smooth manner. Beyond the major shift in our understanding of spacetime which these results entail, they also provide us with a clear window on inherently string-based physics as they are largely insensitive to many detailed aspects of the theory presently beyond our analytic control.

More recently, $D$-branes [5] have provided us with a new physical probe of shortdistance physics in string theory [6]. From this perspective, spacetime is a derived or secondary concept - emerging from the vacuum moduli space of $D$-brane world-volume gauge theories. Determining how the novel string-based properties of spacetime mentioned above appear to $D$-branes is an important and compelling line of study. $D$-branes on orbifold backgrounds have been studied in [7, 8,9, 10, 11, 12, 13] and, as we shall discuss in more detail below, the results match well with string-derived conclusions. $T$-dualities have been studied in works that are too numerous to mention and many important physical implications have been derived. On the contrary, even though the conifold transitions of [4] rely crucially on properties of wrapped $D$-branes [14], and $D$-branes near conifold singularities have been subsequently studied in works such as [15, 16, 17] not as much work has been devoted to using $D$-branes to probe spacetime as it goes through a topology changing transition. In the present work we focus on this issue.

In particular, we study the way in which flop transitions - so-called mild topology changing processes (see [2, 18]) - appear from the viewpoint of $D$-particles. We do this by studying the $D$-particle vacuum moduli space in an orbifold background that admits multiple topologically distinct resolutions. This is the same philosophy we took in [2], the difference being that we now study these rich backgrounds from the perspective of $D$-branes as opposed to fundamental strings. We show that these multiple resolutions are directly reflected in the $D$-brane Yang-Mills vacuum moduli space, and that we can smoothly interpolate from one topology to another by varying the Fayet-Illiopoulos parameters that appear in the $D$-brane gauge theory.

Specifically, in section 2 we review the results of [10] which provide a method for studying $D$-branes on $\mathbb{C}^{3} / \Gamma$, where $\Gamma$ is some finite abelian group. In section 3 , we recall 
the novel way in which $D$-branes - at least in simple examples — barely avoid the nongeometrical phases [2,3] probed by weakly coupled strings, as predicted by the arguments given in [19]. In section 4, we reiterate the point made in [10] that for isolated singularities in $\mathbb{C}^{3}$, the smallest choice for $\Gamma$ giving rise to flop transitions is the calculationally burdensome case of $\mathbb{Z}_{11}$. But we also note the well known fact, as we stressed in [20], that if one foregoes the requirement of isolated singularities, then the more manageable case of $\Gamma=\mathbb{Z}_{2} \times \mathbb{Z}_{2}$ is sufficiently rich to admit distinct resolutions differing by flops. We then carry out the analysis in this example, and explicitly show that the $D$-brane vacuum moduli space incorporates these flop transitions, thereby showing that this novel piece of string physics has a direct $D$-brane counterpart. In section 5 we offer some conclusions.

After this work was completed, a paper by Muto [21] appeared which took up the challenge of [10] and claims to have successfully worked out the $\mathbb{Z}_{11}$ case.

\section{D-branes on $\mathbb{C}^{3} / \Gamma$}

As in [10] we study a single $D$-brane in type II string theory rolling around on $\mathbb{C}^{3} / \Gamma$. In the $d=4$ 'non-compact' dimensions, this has $\mathcal{N}=2$ supersymmetry in the closed string sector, while in the open string sector it has $\mathcal{N}=1$ supersymmetry. We realize the latter by working with an $\mathcal{N}=4 d=4$ theory of $|\Gamma| D$-branes on the $\mathbb{C}^{3}$ covering space, and then projecting to $\mathbb{C}^{3} / \Gamma$. This projection is defined by making two choices: the action of $\Gamma$ on $\mathbb{C}^{3}$ and the action of $\Gamma$ on the Chan-Paton factors of the $U(|\Gamma|)$ gauge group. In order to project to a single $D$-brane in the quotient theory, we should take the latter action of $\Gamma$ to be the regular representation. The only requirement we impose on the former action is that it lie in $S U(3)$ so that at least a single supersymmetry is preserved in passing to the quotient space.

To find the fields in the quotient theory, we identify those which are invariant under these combined actions. Explicitly, if we let $g \in \Gamma$, and denote its actions on $\mathbb{C}^{3}$ and on the Chan-Paton factors by $R(g)$ and $S(g)$ respectively, we can determine the surviving fields as follows: The components of gauge fields $A$, represented by $U(|\Gamma|)$ matrices with indices along the brane world volume, survive if

$$
A_{i j}=\left(S(g) A S(g)^{-1}\right)_{i j}
$$

The scalar fields living on the brane world-volume - again originating as $U(|\Gamma|)$ matrices $X$ - survive if

$$
X_{i j}^{\alpha}=\left(R(g) S(g) X^{\alpha} S(g)^{-1}\right)_{i j}
$$


where $R(g)$ only acts non-trivially in the $\mathbb{C}^{3}$ directions.

After solving these projection constraints, we substitute the surviving fields into the dimensional reduction onto the brane world volume of the covering space $\mathcal{N}=4$ superYang-Mills theory, augmented by the Fayet-Illiopoulos parameters discussed in [7]. The vacuum moduli space of this theory — which we identify with the internal part of spacetime as probed by the $D$-brane - arises from solving the $F$ and $D$-flatness conditions of this supersymmetric gauge theory. The $F$-flatness constraints come from the superpotential $W=\operatorname{Tr}\left[X^{1}, X^{2}\right] X^{3}$ whose minimization $\partial W / \partial X_{i j}^{\alpha}$ yields the equations

$$
\left[X^{\alpha}, X^{\beta}\right]=0
$$

The $D$-flatness conditions arise from the unbroken $U(1)$ gauge symmetries that survive the separating of the $D$-branes and are of the usual form

$$
\sum q_{(k) \alpha}^{\beta}\left|X_{\beta}^{\alpha}\right|^{2}-\zeta_{k}=0
$$

where $q_{(k) \alpha}^{\beta}$ is the charge of $X_{\beta}^{\alpha}$ under the $k^{\text {th }} U(1)$ and $\beta$ runs over the matrix indices of all surviving components of $X^{\alpha}$. For ease of notation, we shall temporarily call these surviving components $x_{1}, x_{2}, \ldots, x_{3|\Gamma|}$ and we shall call the $|\Gamma|-1$ by $|\Gamma|+2$ matrix containing these charges $V$.

In [10], a convenient method for solving these vacuum configuration constraints was introduced, making use of toric geometry to phrase them all in a single unified framework. Specifically, the $F$-flatness conditions are explicitly solved for $2|\Gamma|-2$ of the $x_{i}$ in terms of the remaining $|\Gamma|+2$ variables. These relations can be summarized by writing

$$
x_{i}=\prod_{j=1}^{|\Gamma|+2} x_{j}^{a_{i j}}
$$

where we assume that the $x$ 's are arranged so that the first $|\Gamma|+2$ are the independent variables. We then arrange this data in a $3|\Gamma|$ by $|\Gamma|+2$ matrix $A$ whose entries are the $a_{i j}$. This matrix encodes the 'M-lattice' data (in the language of [2] for example) of the toric variety $\mathcal{T}$ of the scalar fields that solve the superpotential constraints.

This takes us part way toward the vacuum moduli space $\mathcal{V}$ - what remains is to impose the $D$-term constraints. Now, as in (2.4), $D$-term constraints naturally take the form of symplectic quotients: we impose a $\mathbb{C}^{*}$ constraint by enforcing moment map conditions and then modding-out by a $U(1)$ symmetries. In order to impose these conditions 
in a manner that efficiently meshes with our $F$-flatness constraints, we are therefore led to rephrase the latter in a symplectic framework as well. This is straightforward to do. From the matrix $A$ we construct a 'dual' $|\Gamma|+2$ by $k$ matrix $T$ whose columns span the dual cone to that spanned by the rows of $A$. (We are not aware of a general expression for $k$; in examples, it is calculated by brute force enumeration of the dual cone.) The $k$ columns of $T$ are associated with $k$ homogeneous coordinates $p_{0}, p_{1}, \ldots, p_{k-1}$ as discussed, for example, in [2]. The transpose of the kernel of $T$, as shown in [22] gives the charge matrix $Q$ for $\mathcal{T}$ realized as a symplectic quotient. All that remains, therefore, is to augment $Q$ with the additional symplectic quotients that enforce the $D$-term constraints. In [10] it was shown that this is accomplished as follows.

We introduce a $|\Gamma|+2$ by $k$ matrix $U$ that satisfies $T U^{t}=\mathbf{1}$. Then, the $D$-term charges of the fields in this dual representation are given by the $|\Gamma|-1$ by $k$ matrix $V U$. Thus, we can combine the $F$ and $D$-flatness conditions by concatenating the matrix $Q$ and the matrix $V U$ into a $(k-3)$ by $k$ matrix $Q^{\text {total }}$. The vacuum moduli space $\mathcal{V}$ is thus realized by the symplectic quotient of $\mathbb{C}^{k}$ by $U(1)^{k-3}$, with the latter action being determined by $Q^{\text {total }}$.

This is almost, but not quite, the whole story. The symplectic quotient just alluded to involves $k-3$ moment maps whose precise form also relies on the values of FayetIlliopoulos parameters coming from the $D$-terms. The choice of these parameters fills out the definition of the model and allows complete specification of $\mathcal{V}$.

\section{An Example}

An example will make this discussion more clear as well as provide a jumping off point for our discussion of flops in the next section. We choose the simplest example of $\Gamma=\mathbb{Z}_{3}$, with action on $\mathbb{C}^{3}$ given by

$$
R(g):(X, Y, Z) \rightarrow\left(\omega^{-1} X, \omega^{-1} Y, \omega^{-1} Z\right)
$$

where $g$ generates $\mathbb{Z}_{3}, \omega$ is a nontrivial cube-root of unity, and $S(g)$ is taken to be the regular representation. Then, as originally discussed in [10], if we call the 9 variables $x_{1}, \ldots, x_{9}=$ $x_{0}, x_{1}, x_{2}, y_{0}, y_{1}, y_{2}, z_{0}, z_{1}, z_{2}$ (where the notation $x_{0,1,2}$ denotes the three components of the coordinate $X$ - after it is promoted to a Yang-Mills matrix — which survive the projection, etc.) we have the matrix $A$ given by the following integer entries 


$\begin{array}{cccccc} & x_{0} & y_{0} & z_{0} & z_{1} & z_{2} \\ x_{0} & 1 & 0 & 0 & 0 & 0 \\ x_{1} & 1 & 0 & -1 & 1 & 0 \\ x_{2} & 1 & 0 & -1 & 0 & 1 \\ y_{0} & 0 & 1 & 0 & 0 & 0 \\ y_{1} & 0 & 1 & -1 & 1 & 0 \\ y_{2} & 0 & 1 & -1 & 0 & 1 \\ z_{0} & 0 & 0 & 1 & 0 & 0 \\ z_{1} & 0 & 0 & 0 & 1 & 0 \\ z_{2} & 0 & 0 & 0 & 0 & 1\end{array}$

where we label the columns by the chosen set of independent fields. Its dual $T$ is

$$
T=\left(\begin{array}{llllll}
1 & 0 & 0 & 1 & 0 & 0 \\
0 & 1 & 0 & 1 & 0 & 0 \\
0 & 0 & 1 & 1 & 0 & 0 \\
0 & 0 & 1 & 0 & 1 & 0 \\
0 & 0 & 1 & 0 & 0 & 1
\end{array}\right)
$$

which has kernel given by the transpose of

$$
Q=\left(\begin{array}{llllll}
1 & 1 & 1 & -1 & -1 & -1
\end{array}\right)
$$

A suitable choice for the matrix $U$ is

$$
U=\left(\begin{array}{cccccc}
0 & -1 & -1 & 1 & 1 & 1 \\
0 & 1 & 0 & 0 & 0 & 0 \\
0 & 0 & 1 & 0 & -1 & -1 \\
0 & 0 & 0 & 0 & 1 & 0 \\
0 & 0 & 0 & 0 & 0 & 1
\end{array}\right)
$$

which yields the matrix

$$
Q^{\text {total }}=\left(\begin{array}{cccccc}
1 & 1 & 1 & -1 & -1 & -1 \\
0 & 0 & 0 & 0 & -1 & 1 \\
0 & 0 & 0 & 1 & 0 & -1
\end{array}\right)
$$

As in the last section, from $Q^{\text {total }}$ and a specification of the Fayet-Illiopoulos parameters we can determine the precise form of $\mathcal{V}$. However, even without specifying values for the Fayet-Illiopoulos parameters (which we will do momentarily) we can use $Q^{\text {total }}$ to determine the 'N-lattice' data of $\mathcal{V}$ by taking the kernel of $Q^{\text {total }}$ and eliminating all redundant rows. Doing so gives us the point set in $\mathbb{R}^{3}$ :

$$
\begin{aligned}
& e_{1}=(1,0,0) \\
& e_{2}=(0,1,0) \\
& e_{3}=(0,0,1) \\
& e_{4}=(-1,-1,3)
\end{aligned},
$$


which we immediately recognize as the toric data for resolving $\mathbb{C}^{3} / \mathbb{Z}_{3}$. Thus, strikingly, the $D$-brane vacuum moduli space $\mathcal{V}$ aligns with the internal space on which we set the $D$-brane moving.

A more subtle test of this correspondence requires that we also include the FayetIlliopoulos terms, as we now do. As in [10], a convenient way to do this is to augment $Q^{\text {total }}$ by an additional column which encodes the Fayet-Illiopoulos term for each of the corresponding rows. For this example this yields

$$
\left(\begin{array}{ccccccc}
1 & 1 & 1 & -1 & -1 & -1 & 0 \\
0 & 0 & 0 & 0 & -1 & 1 & \zeta_{1} \\
0 & 0 & 0 & 1 & 0 & -1 & \zeta_{2}
\end{array}\right)
$$

Notice that the Fayet-Illiopoulos term vanishes for the upper row since this constraint originated from an $F$-flatness condition in our original model. This, in fact, is the crucial point. It means that the symplectic quotient associated with $Q^{\text {total }}$ is not generic since the moment maps do not have generic arguments. This, in turn, constrains the regions of the phases-picture of [2,3] that are physically accessible by the D-brane. Lets recall what this observation implies in this simple example.

Doing so requires that we allow $\zeta_{1,2}$ to freely run over all (real) values, and for each such possibility we must carry out the symplectic quotient to determine $\mathcal{V}$. This is easier done than said: a choice of sign for each of $\zeta_{1,2}$, via the last two rows of $Q^{\text {total }}$, ensures that two of the last three $p$ variables cannot vanish. We then eliminate these two variables by doing row operations that eliminate them from the first row. For example, assume that $\zeta_{1,2}$ are both positive. Then we see that $p_{3}$ and $p_{5}$ cannot vanish and we eliminate them by adding row three and two times row two to row one. This yields the matrix

$$
\left(\begin{array}{ccccccc}
1 & 1 & 1 & 0 & -3 & 0 & 2 \zeta_{1}+\zeta_{2} \\
0 & 0 & 0 & 0 & -1 & 1 & \zeta_{1} \\
0 & 0 & 0 & 1 & -1 & 0 & \zeta_{1}+\zeta_{2}
\end{array}\right)
$$

The important thing to note is that the Fayet-Illiopoulos term in the first row, $2 \zeta_{1}+\zeta_{2}$, is of fixed (positive) sign. This ensures that we are in the blown up phase of $\mathbb{C}^{3} / \mathbb{Z}_{3}$. A similar thing holds true for all other choices of signs of $\zeta_{1,2}$, as the reader can easily confirm. In this simple example, then, carrying out all but $h^{11}=1$ of the symplectic quotients the ones which manifestly constrain some of the homogeneous coordinates to lie in $\mathbb{C}^{*}$ uniquely fixes the sign of the remaining Fayet-Illiopoulos parameters. And moreover, the constraint keeps us in the fully resolved geometric phase of the model, not allowing us 
to enter the analog of the Landau-Ginsburg phase. This is in precise keeping with the observation of [19] that the results of [23] can be used to argue that only geometric phases survive to the strong coupling limit. As $D$-branes are the most relevant degrees of freedom in this limit, we anticipate that they should only probe geometric phases as well, and this is precisely what occurs in this example.

The question we now pose is whether $D$-branes necessarily probe all possible geometric phases of a model. This example, and the others studied in [10] are too simple to address this issue, as they each have a unique geometric phase region, and hence we must undertake further analysis.

\section{D-brane Flops}

As mentioned at the end of [10], the simplest cyclic quotient singularity of $\mathbb{C}^{3}$ that admits flops is $\mathbb{Z}_{11}$, a prohibitively large number. So rather than examining that case, let's leave the domain of isolated cyclic quotients and consider the case of $\Gamma=\mathbb{Z}_{2} \times \mathbb{Z}_{2}$. As is well known, $\mathbb{C}^{3} /\left(\mathbb{Z}_{2} \times \mathbb{Z}_{2}\right)$ does admit distinct flop related resolutions. And as mentioned in [20] and now explicitly shown, unlike the case reviewed above, there is freedom in the sign of the Fayet-Illiopoulos parameters that arise from reducing $Q^{\text {total }}$ by eliminating homogeneous variables forced to lie in $\mathbb{C}^{*}$. As we shall see, this freedom coincides with the ability to flop rational curves in the $D$-brane vacuum moduli space $\mathcal{V}$.

One complication of this quotient is that the resulting singularity is not isolated. For the analysis we perform here, this hardly changes our procedure. However, if we wanted to fully express the complexified conformal field theory blow up modes in terms of the Yang-Mills gauge theory parameters, we would have to work harder than we do here. This is an issue to which we hope to return.

We choose our $\mathbb{Z}_{2} \times \mathbb{Z}_{2}$ generators $g_{1}, g_{2}$ to act on $\mathbb{C}^{3}$ according to

$$
\begin{aligned}
& g_{1}:(X, Y, Z) \rightarrow(-X,-Y, Z) \\
& g_{2}:(X, Y, Z) \rightarrow(-X, Y,-Z)
\end{aligned}
$$

Then, after diagonalizing, the regular representation is given by

$$
S\left(g_{1}\right)=\left(\begin{array}{llll}
1 & & & \\
& 1 & & \\
& & -1 & \\
& & & -1
\end{array}\right)
$$


and

$$
S\left(g_{2}\right)=\left(\begin{array}{llll}
1 & & & \\
& -1 & & \\
& & 1 & \\
& & & -1
\end{array}\right)
$$

It is now straightforward to do the projections and realize that the surviving fields are:

$$
\begin{gathered}
\left(x_{1}, x_{2}, x_{3}, x_{4}\right)=\left(X_{14}, X_{23}, X_{32}, X_{41}\right) \\
\left(y_{1}, y_{2}, y_{3}, y_{4}\right)=\left(Y_{13}, Y_{24}, Y_{31}, Y_{42}\right) \\
\left(z_{1}, z_{2}, z_{3}, z_{4}\right)=\left(Z_{12}, Z_{21}, Z_{34}, Z_{43}\right)
\end{gathered}
$$

where the right-hand-side denotes, again, the components of the matrices arising from the Yang-Mills description (whose spacetime index is in the direction of the coordinates $X, Y$ or $Z$ ).

A little algebra now shows that the $F$-flatness constraints are solved by the relations encoded in the matrix $A$ :

$\begin{array}{ccccccc} & x_{1} & x_{2} & x_{3} & y_{1} & y_{2} & z_{1} \\ x_{1} & 1 & 0 & 0 & 0 & 0 & 0 \\ x_{2} & 0 & 1 & 0 & 0 & 0 & 0 \\ x_{3} & 0 & 0 & 1 & 0 & 0 & 0 \\ x_{4} & -1 & 1 & 1 & 0 & 0 & 0 \\ y_{1} & 0 & 0 & 0 & 1 & 0 & 0 \\ y_{2} & 0 & 0 & 0 & 0 & 1 & 0 \\ y_{3} & -1 & 0 & 1 & 0 & 1 & 0 \\ y_{4} & -1 & 0 & 1 & 1 & 0 & 0 \\ z_{1} & 0 & 0 & 0 & 0 & 0 & 1 \\ z_{2} & -1 & 1 & 0 & -1 & 1 & 1 \\ z_{3} & 0 & 0 & 0 & -1 & 1 & 1 \\ z_{4} & -1 & 1 & 0 & 0 & 0 & 1\end{array}$

Following the procedure discussed in section 2, we now calculate the dual matrix $T$ :

$$
T=\left(\begin{array}{lllllllll}
1 & 1 & 1 & 0 & 0 & 0 & 0 & 0 & 0 \\
0 & 1 & 1 & 1 & 0 & 0 & 0 & 0 & 0 \\
1 & 1 & 0 & 0 & 1 & 0 & 0 & 0 & 0 \\
0 & 0 & 1 & 0 & 0 & 1 & 0 & 1 & 0 \\
0 & 0 & 1 & 0 & 0 & 1 & 0 & 0 & 1 \\
1 & 0 & 0 & 0 & 0 & 0 & 1 & 1 & 0
\end{array}\right)
$$


The kernel of this matrix is

$$
Q=\left(\begin{array}{ccccccccc}
0 & 1 & -1 & 0 & -1 & 1 & 0 & 0 & 0 \\
-1 & 1 & 0 & -1 & 0 & 0 & 1 & 0 & 0 \\
-1 & 2 & -1 & -1 & -1 & 0 & 0 & 1 & 1
\end{array}\right)
$$

This gives us our standard toric geometry (equivalently, linear sigma model) construction of what we have been calling $\mathcal{T}$ - the locus of the fields meeting the superpotential constraints. We now need to include the $D$-term constraints. To do so, we follow the procedure given earlier and calculate the matrix $U$ to be

$$
U=\left(\begin{array}{ccccccccc}
0 & 1 & 0 & -1 & -1 & 0 & 0 & 0 & 0 \\
0 & 0 & 0 & 1 & 0 & 0 & 0 & 0 & 0 \\
0 & 0 & 0 & 0 & 1 & 0 & 0 & 0 & 0 \\
-1 & 1 & 0 & -1 & 0 & 0 & 0 & 1 & 0 \\
1 & -2 & 1 & 1 & 1 & 0 & 0 & -1 & 0 \\
1 & -1 & 0 & 1 & 0 & 0 & 0 & 0 & 0
\end{array}\right)
$$

Together with the $U(1)^{3}$ charge matrix

$$
V=\left(\begin{array}{cccccc}
1 & 0 & 0 & 1 & 0 & 1 \\
0 & 1 & -1 & 0 & 1 & -1 \\
0 & -1 & 1 & -1 & 0 & 0
\end{array}\right)
$$

we learn that our 9 homogeneous variables, $p_{0}, p_{1}, \ldots, p_{8}$ have charges $V U$ given by

$$
V U=\left(\begin{array}{ccccccccc}
0 & 1 & 0 & -1 & -1 & 0 & 0 & 1 & 0 \\
0 & -1 & 1 & 1 & 0 & 0 & 0 & -1 & 0 \\
1 & -1 & 0 & 0 & 1 & 0 & 0 & -1 & 0
\end{array}\right) .
$$

Concatenating this with the charge matrix $Q$ yields

$$
Q^{\text {total }}=\left(\begin{array}{ccccccccc}
0 & 1 & -1 & 0 & -1 & 1 & 0 & 0 & 0 \\
-1 & 1 & 0 & -1 & 0 & 0 & 1 & 0 & 0 \\
-1 & 2 & -1 & -1 & -1 & 0 & 0 & 1 & 1 \\
0 & 1 & 0 & -1 & -1 & 0 & 0 & 1 & 0 \\
0 & -1 & 1 & 1 & 0 & 0 & 0 & -1 & 0 \\
1 & -1 & 0 & 0 & 1 & 0 & 0 & -1 & 0
\end{array}\right)
$$

Now, before including the Fayet-Illiopoulos terms, we calculate the kernel of this charge matrix, and find the (distinct) points in $\mathbb{R}^{3}$

$$
\begin{aligned}
& e_{1}=(1,0,0) \\
& e_{2}=(0,1,0) \\
& e_{3}=(0,0,1) \\
& e_{4}=(2,1,-2) \\
& e_{5}=(0,-1,2) \\
& e_{6}=(1,1,-1)
\end{aligned}
$$


It is not hard to see that this is the toric point set for resolving $\mathbb{C}^{3} /\left(\mathbb{Z}_{2} \times \mathbb{Z}_{2}\right)$. Explicitly, the toric data for $\mathbb{C}^{3} /\left(\mathbb{Z}_{2} \times \mathbb{Z}_{2}\right)$ can be found by augmenting the $\mathbb{Z}^{\oplus 3}$ lattice (generated by the standard basis vectors $e_{1}, e_{2}, e_{3}$ over $\left.\mathbb{Z}\right)$ by the fractional points $(1 / 2,1 / 2,0)$ and $(1 / 2,0,1 / 2)$. In other words, we build a new lattice whose generators are these points, together with those generating the original $\mathbb{Z}^{\oplus 3}$ lattice. The reason we do this, as explained for instance in [22], is that, in general, including fractional points $\left(h_{1}, \ldots, h_{n}\right)$ in the toric $N$ lattice $\mathbb{Z}^{\oplus n}$ yields the one-parameter group action on $\left(\mathbb{C}^{*}\right)^{n}$ with coordinates $\left(\rho_{1}, \ldots, \rho_{n}\right)$ of the form

$$
\left(\rho_{1}, \ldots, \rho_{n}\right) \rightarrow\left(e^{2 \pi i h_{1}} \rho_{1}, \ldots, e^{2 \pi i h_{n}} \rho_{n}\right) .
$$

For $h_{i}=a_{i} / n$ with $a_{i} \in \mathbb{Z}$, we see that this enforces a $\mathbb{Z}_{n}$ identification, that is, a $\mathbb{Z}_{n}$ orbifold. Thus, adding the points $(1 / 2,1 / 2,0)$ and $(1 / 2,0,1 / 2)$ enforces the identifications on $\mathbb{C}^{3}$ given by the action of $g_{1}$ and $g_{2}$. Note also that we necessarily must also include $(0,1 / 2,1 / 2)$ as this identification arises from $g_{1} g_{2}$ (and, equivalently, it is also a lattice point in our augmented lattice). To put this in a more recognizable form, we can now change basis to restore integrality in our augmented lattice. Namely, with

$$
\begin{aligned}
e_{1} & =(1,0,0) \\
e_{2} & =(0,1,0) \\
e_{3} & =(0,0,1) \\
e_{4}^{\prime} & =(1 / 2,1 / 2,0) \\
e_{5}^{\prime} & =(1 / 2,0,1 / 2) \\
e_{6}^{\prime} & =(0,1 / 2,1 / 2)
\end{aligned}
$$

we can change basis by expressing all vectors in terms of $e_{2}, e_{4}^{\prime}, e_{5}^{\prime}$, which we now call $(0,1,0),(0,0,1),(1,0,0)$, yielding $e_{1}=(0,-1,2), e_{3}=(2,1,-2)$ and $e_{6}^{\prime}=(1,1,-1)$. We recognize that this list of six points in $\mathbb{R}^{3}$ exactly matches the list of points in (4.15), thus establishing that we have recovered - from our $D$-brane vacuum moduli space - the toric data for resolving our internal $\mathbb{C}^{3} /\left(\mathbb{Z}_{2} \times \mathbb{Z}_{2}\right)$ component of space.

For the final step we need to include the Fayet-Illiopoulos parameters. Doing so yields

$$
Q^{\text {total }}=\left(\begin{array}{cccccccccc}
0 & 1 & -1 & 0 & -1 & 1 & 0 & 0 & 0 & 0 \\
-1 & 1 & 0 & -1 & 0 & 0 & 1 & 0 & 0 & 0 \\
-1 & 2 & -1 & -1 & -1 & 0 & 0 & 1 & 1 & 0 \\
0 & 1 & 0 & -1 & -1 & 0 & 0 & 1 & 0 & \zeta_{1} \\
0 & -1 & 1 & 1 & 0 & 0 & 0 & -1 & 0 & \zeta_{2} \\
1 & -1 & 0 & 0 & 1 & 0 & 0 & -1 & 0 & \zeta_{3}
\end{array}\right)
$$


Then, by adding the fourth row to each of rows five and six this becomes

$$
\left(\begin{array}{cccccccccc}
0 & 1 & -1 & 0 & -1 & 1 & 0 & 0 & 0 & 0 \\
-1 & 1 & 0 & -1 & 0 & 0 & 1 & 0 & 0 & 0 \\
-1 & 2 & -1 & -1 & -1 & 0 & 0 & 1 & 1 & 0 \\
0 & 1 & 0 & -1 & -1 & 0 & 0 & 1 & 0 & \zeta_{1} \\
0 & 0 & 1 & 0 & -1 & 0 & 0 & 0 & 0 & \zeta_{1}+\zeta_{2} \\
1 & 0 & 0 & -1 & 0 & 0 & 0 & 0 & 0 & \zeta_{1}+\zeta_{3}
\end{array}\right)
$$

which is a particularly nice form since it immediately allows us to identify homogeneous coordinates lying in $\mathbb{C}^{*}$ by the signs of $\zeta_{1}+\zeta_{2}$ and $\zeta_{1}+\zeta_{3}$. In fact, for definiteness let's assume that we examine the part of moduli space in which each of these combinations of $\zeta$ 's is positive. This means we must eliminate $p_{0}$ and $p_{2}$. We can again accomplish this by row operations taking us to

$$
\left(\begin{array}{cccccccccc}
0 & 1 & 0 & 0 & -2 & 1 & 0 & 0 & 0 & \zeta_{1}+\zeta_{2} \\
0 & 1 & 0 & -2 & 0 & 0 & 1 & 0 & 0 & \zeta_{1}+\zeta_{3} \\
0 & 0 & 0 & 0 & 0 & 0 & 0 & -1 & 1 & \zeta_{2}+\zeta_{3} \\
0 & 1 & 0 & -1 & -1 & 0 & 0 & 1 & 0 & \zeta_{1} \\
0 & 0 & 1 & 0 & -1 & 0 & 0 & 0 & 0 & \zeta_{1}+\zeta_{2} \\
1 & 0 & 0 & -1 & 0 & 0 & 0 & 0 & 0 & \zeta_{1}+\zeta_{3}
\end{array}\right),
$$

where we have also taken the liberty of doing invertible row operations that simplify row 3 , as shown. This is an even nicer form since if we further assume, for instance, that $\zeta_{2}+\zeta_{3}$ is positive, the third row allows us to eliminate $p_{8}$ (which does not appear in any other constraint equation). Putting all this together takes us to the reduced form of a symplectic quotient of $\mathbb{C}^{6}$ (the variables $\left.p_{1}, p_{3}, p_{4}, p_{5}, p_{6}, p_{7}\right)$ by the $U(1)^{3}$ action given by

$$
Q^{\text {reduced }}=\left(\begin{array}{ccccccc}
1 & 0 & -2 & 1 & 0 & 0 & \zeta_{1}+\zeta_{2} \\
1 & -2 & 0 & 0 & 1 & 0 & \zeta_{2}+\zeta_{3} \\
1 & -1 & -1 & 0 & 0 & 1 & \zeta_{1}
\end{array}\right)
$$

in which we have now used rows 3,5 , and 6 of (4.20), together with our assumption on the $\zeta$ 's, to eliminate the columns associated with $p_{0}, p_{2}$, and $p_{8}$.

The key point to notice is that the part of moduli space we are focusing on, $\zeta_{1}+\zeta_{2}>$ $0, \zeta_{1}+\zeta_{3}>0, \zeta_{2}+\zeta_{3}>0$, does not constrain the sign of $\zeta_{1}$ in the third row of (4.21) above. That is, unlike the case of $\mathbb{Z}_{3}$ reviewed earlier, when we fix a region of moduli space to eliminate constrained homogeneous variables in this context, it does not uniquely constrain the sign of the resulting combination of Fayet-Illiopoulos parameters.

The reason this is of relevance comes from the toric interpretation of the last line in (4.21). This equation is

$$
\left|p_{1}\right|^{2}+\left|p_{7}\right|^{2}-\left|p_{3}\right|^{2}-\left|p_{4}\right|^{2}=\zeta_{1}
$$


which yields a $\mathbb{P}^{1}$ that is flopped as the sign of $\zeta_{1}$ changes. In toric terms, this equation is equivalent to the data of four vectors in $\mathbb{R}^{3}, \vec{u}_{2}, \vec{u}_{7}, \vec{u}_{3}, \vec{u}_{4}$ satisfying

$$
\vec{u}_{1}+\vec{u}_{7}=\vec{u}_{3}+\vec{u}_{4}
$$

as shown in figure 1.

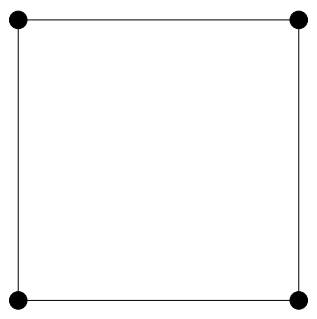

Fig. 1: The four toric vectors $\vec{u}_{i}$ in $\mathbb{R}^{3}$ yielding a (double-point) singularity.

As written, these toric points yield an ordinary double point. We can resolve this singularity by triangulating the point set, but, as we illustrate in figure 2 , this can be done in two ways, differing by a flop of a rational curve. From a symplectic standpoint, it is the sign of $\zeta_{1}$ that determines which of these two resolutions is used.

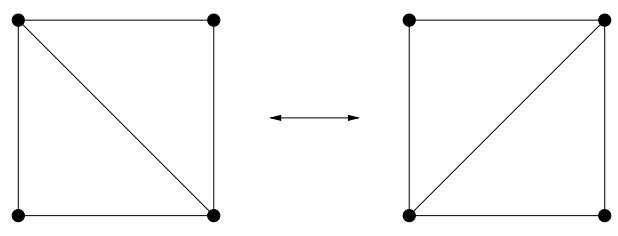

Fig. 2: The two small resolutions of the singularity.

And so we see that the freedom of sign in $\zeta_{1}$ corresponds to the freedom of flopping a rational curve in the $D$-brane vacuum moduli space. In the full context of $\mathbb{C}^{3} /\left(\mathbb{Z}_{2} \times \mathbb{Z}_{2}\right)$ we see these flops in the toric diagram of the points in (4.21) of which we show one example in figure 3. 


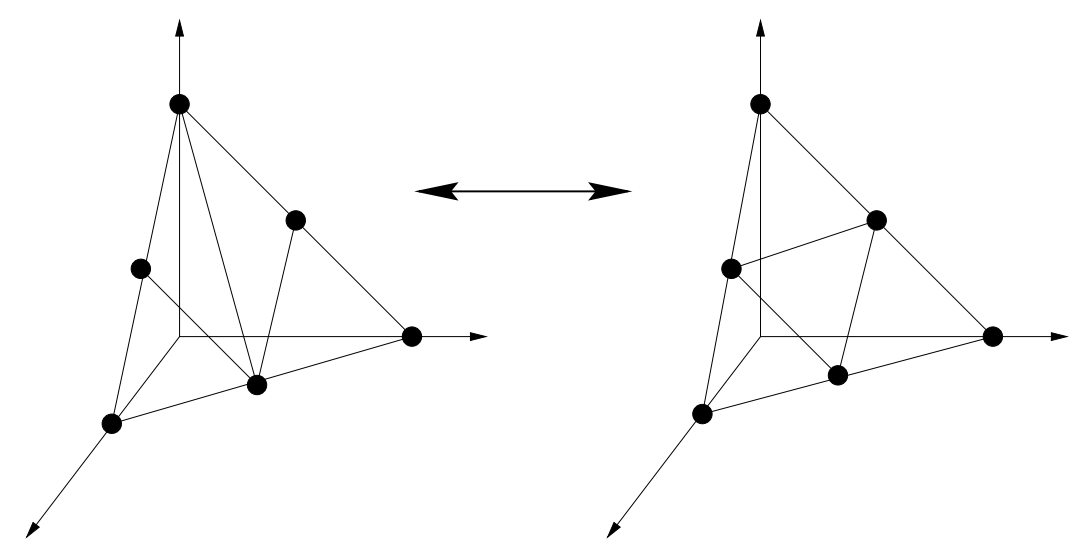

Fig. 3: An example of a flop transition in resolving $\mathbb{C}^{3} /\left(\mathbb{Z}_{2} \times \mathbb{Z}_{2}\right)$.

\section{Conclusions}

The arguments presented here are closer in spirit to those of [2] than to those of [3] because they establish topology change from a global as opposed to a local perspective. We have shown that the $D$-brane vacuum moduli space does have regions related by flop transition, and then, by virtue of supersymmetry, we know that singularities are at least of complex codimension 1, ensuring smooth passage from one region to another. Nevertheless, it would be very interesting to understand the $D$-brane analog of the analysis of [3] as this would give insight into the microscopic features underlying topology change according to these non-perturbative string degrees of freedom.

Furthermore, it would be very interesting to fully understand the map between the complexified nonlinear sigma model variables and the parameters of the $D$-brane gauge theory (which, in the non-isolated case, apparently requires additional gauge theory parameters). This would allow direct comparison of quantum volumes as probed by strings and by $D$-branes, and, for instance, could be used to study the local dynamics around the more drastic conifold topology changing processes as well.

\section{Acknowledgments}

The author gratefully acknowledges the support of a National Young Investigator Award, the Department of Energy, and an Alfred P. Sloan Foundation Fellowship. 


\section{References}

[1] L. Dixon, J. A. Harvey, C. Vafa, and E. Witten, "Strings on Orbifolds, I, II" Nucl. Phys. B261 (1985) 678; Nucl. Phys. B274 (1986) 285.

[2] P. S. Aspinwall, B. R. Greene and D. R. Morrison, "Calabi-Yau Moduli Space, Mirror Manifolds and Spacetime Topology Change in String Theory," Nucl. Phys. B416 (1994) 414; hep-th/9309097.

[3] E. Witten, "Phases of $N=2$ Theories In Two Dimensions," Nucl. Phys. B403 (1993) 159; hep-th/9301042.

[4] B. R. Greene, D. R. Morrison, A. Strominger, "Black Hole Condensation and the Unification of String Vacua", Nucl. Phys. B451 (1995) 109.

[5] J. Polchinski, "Dirichlet-Branes and Ramond-Ramond Charges", Phys. Rev. Lett. 75 (1995) 4724-4727.

[6] M. R. Douglas, D. Kabat, P. Pouliot, S. H. Shenker, "D-branes and Short Distances in String Theory", Nucl.Phys. B485 (1997) 85-127.

[7] M. R. Douglas and G. Moore, "D-Branes, Quivers, and ALE Instantons," hepth/9603167.

[8] C. Johnson and R. Myers, "Aspects of Type IIB Theory on ALE Spaces," hepth/9610140.

[9] J. Polchinski, "Tensors from K3 Orientifolds," hep-th/9606165.

[10] M. R. Douglas, B. R. Greene, D. R. Morrison, "Orbifold Resolution by D-Branes", hep-th/9704151.

[11] M. R. Douglas and B. R. Greene, "Metrics on D-brane Orbifolds", hep-th/9707214.

[12] M. R. Douglas, H. Ooguri and S. H. Shenker, "Issues in M(atrix) Theory Compactification," hep-th/9702203.

[13] M. R. Douglas, A. Kato, H. Ooguri, "D-brane Actions on Kahler Manifolds", hepth/9708012.

[14] A. Strominger, "Massless Black Holes and Conifolds in String Theory", Nucl. Phys. B451 (1995) 96.

[15] K. Hori, H. Ooguri, C. Vafa, "Non-Abelian Conifold Transitions and N=4 Dualities in Three Dimensions", hep-th/9705220.

[16] E. Gava, T. Jayaraman, K. S. Narain, M. H. Sarmadi, "D-branes and the Conifold Singularity", Phys.Lett. B388 (1996) 29.

[17] D. P. Jatkar, B. Peeters, "String Theory near a Conifold Singularity", Phys.Lett. B362 (1995) 73.

[18] B.R. Greene, "String Theory on Calabi-Yau Manifolds", Proceedings of TASI-96, hepth/9702155.

[19] E. Witten, "Phase Transitions In M-Theory And F-Theory," Nucl. Phys. B471 (1996) 195; hep-th/9603150. 
[20] B.R. Greene, Talk at Strings '97, Amsterdam, 1997.

[21] T. Muto, "D-branes on Orbifolds and Topology Change", hep-th/9711090.

[22] P. S. Aspinwall and B. R. Greene, "On the Geometric Interpretation of $N=2$ Superconformal Theories," Nucl. Phys. B437 (1995) 205; hep-th/9409110.

[23] P. S. Aspinwall, B. R. Greene and D. R. Morrison, "Measuring Small Distances in $N=2$ Sigma Models," Nucl. Phys. B420 (1994) 184; hep-th/9311042. 